\title{
Revisión de la versión adaptada del Cuestionario de Expectativas hacia el Alcohol (AEQ) para jóvenes universitarios
}

\author{
Martha Leticia Salazar Garza', Silvia Jazmín Ortiz Noriega', Ma. de los Ángeles Vacio Muro' \\ 'Departamento de Psicología, Centro de Ciencias Sociales y Humanidades, Universidad Autónoma de Aguascalientes, México
}

\section{RESUMEN}

Introducción: el Cuestionario de Expectativas hacia el Alcohol (AEQ) es empleado para medir las creencias de los individuos sobre el consumo de esta sustancia. En México se cuenta con una adaptación del instrumento, aunque al revisarlo se llegó a la conclusión de que algunos ítems no reflejaban las creencias en términos de una relación de causalidad y que las opciones de respuesta presentadas en forma dicotómica reducían la posibilidad de que los estudiantes respondieran de una manera más cercana a su realidad. Objetivo: obtener las características psicométricas de una versión revisada y modificada del AEQ, adaptado previamente en México. Método: se trabajó con una muestra de 510 estudiantes universitarios, siguiendo las normas para el desarrollo y revisión de estudios instrumentales; mediante el empleo de análisis factorial confirmatorio y el análisis de validez concurrente. Resultados: la versión final del instrumento quedó integrada por 26 reactivos, agrupados en siete factores: desinhibición, confianza en sí mismo, reducción de la tensión, relajación, incremento de la sexualidad, interacción social y cambios psicofisiológicos; con índice general de Confiabilidad de .937. Discusión y conclusiones: posiblemente la revisión conceptual y operacional influyó en el reacomodo y la eliminación de reactivos en comparación con la versión adaptada previamente en nuestro país; sin embargo, los análisis muestran que las expectativas positivas guardan una relación con la frecuencia de consumo excesivo de alcohol. Se obtuvo una versión del AEQ más corta, con validez de contenido, factorial y concurrente, y un índice de confiabilidad aceptable para cada dimensión.

\begin{abstract}
Introduction: the Alcohol Expectancy Questionnaire (AEQ) is used to measure the beliefs of individuals about alcohol consumption. In Mexico there is an adaptation of the instrument, although when reviewing it, it was concluded that some items did not reflect beliefs in terms of a causality relationship and that the response options presented in a dichotomous manner reduced the possibility that students respond in a way that is closer to your reality. Objective: obtain the psychometric characteristics of a revised and modified version of the AEQ, previously adapted in Mexico. Method: a sample of 510 university students, following the rules for the development and revision of instrumental studies; through the use of confirmatory factor analysis and concurrent validity analysis. Results: the final version of the instrument was composed of 26 items, in seven factors: disinhibition, self-confidence, tension reduction, relaxation, increased sexuality, social interaction and psychophysiological changes; with a general Reliability index of .937. Discussion and conclusions: possibly the conceptual and operational review influenced the re-arrangement and elimination of reagents in comparison with the previously adapted version in our country. However, the analyzes show that positive expectations have a positive relationship with the frequency of excessive alcohol consumption. A shorter version of the AEQ was obtained, with content validity, factorial and concurrent, and an acceptable reliability index for each dimension.
\end{abstract}

Keywords: alcohol consumption, expectations, AEQ, college students.

Palabras claves: consumo de alcohol, expectativas, $A E Q$, estudiantes universitarios.

\footnotetext{
Autor de correspondencia:

Martha Leticia Salazar Garza. Departamento de Psicología, Universidad Autónoma de Aguascalientes. Av. Universidad núm. 940, Ciudad Universitaria, C.P. 20131, Aguascalientes. Correo electrónico: mlsalazar@correo.uaa.mx

Recibido: 7 de junio de 2017

Aceptado: 02 de abril de 2018

doi: 10.28931/riiad.2018.1.04
} 


\section{INTRODUCCIÓN}

Iniciar el consumo de alcohol a edad temprana aumenta la posibilidad de continuar con su uso en la edad adulta (Ardila \& Herrán, 2008). La ingesta de dicha droga está ligada, entre otras variables, a las expectativas positivas que los jóvenes tienen sobre su uso, sobre todo las relacionadas con la desinhibición social y el enfrentamiento a situaciones difíciles (Leiva, Pereira, \& Lobo, 2010). Es la droga más reportada por consumo inicial y la que ocasiona mayores casos de ingreso en instituciones de salud por problemas graves, debido a su aceptación social y a la baja percepción de riesgo que se tiene sobre su consumo, en especial entre los adolescentes y adultos consumidores; en parte, esto puede ayudar a explicar el aumento en su consumo (Instituto Nacional de Psiquiatría Ramón de la fuente Muñiz [INPRFM], Instituto Nacional de Salud Pública [INSP], Comisión Nacional Contra las Adicciones, \& Secretaría de Salud [SS], 2017; Villatoro et al., 2011).

Por otro lado, se ha considerado que la adolescencia y la juventud son periodos en los cuales se tiene una baja percepción de riesgo, en comparación con la población adulta, lo que lleva a los jóvenes a involucrarse en conductas que perjudican su salud, por disfrutar de la adrenalina que esto causa; tal es el caso del consumo de alcohol. Los jóvenes tienen la creencia de que una vez iniciado el consumo se puede tener el control sobre el mismo (García del Castillo, 2012; Kumate, 2003).

El consumo de alcohol es una acción aceptada y vista como algo común en la vida de los universitarios, pues se relaciona con la interacción social; al generarles sensaciones agradables, pesan más las expectativas y beneficios percibidos (Londoño \& Valencia, 2010), que las posibles consecuencias negativas.

Algunos investigadores explican el consumo de alcohol a través de un modelo, en el cual describen que la ingesta se ve afectada directa e indirectamente por diferentes variables: la frecuencia de asistencia a fiestas y las expectativas negativas (probabilidad de experimentar conductas negativas) afectan directamente el consumo; mientras que el mejoramiento social, el afrontamiento y los amigos bebedores lo influyen de manera indirecta (Aguilar, Aguilar, \& Valencia, 2011).

Monk y Heim (2016) utilizaron el AEQ y encontraron que quienes lo consumen esperan con mayor frecuencia las consecuencias positivas, en comparación con las negativas; mientras que en la población joven (bachilleres y universitarios), las principales expectativas están relacionadas con la diversión y los aspectos sociales.

Otro instrumento que mide las expectativas es el Cuestionario de Expectativas de Resultado de Consumo de Alcohol (CERCA), basado en la teoría del aprendizaje social de Bandura; cuenta con tres dimensiones: Habilidades sociales, Disminución del estrés y Facilitación social; está redactado en sentido afirmativo y da opciones de respuesta en formato tipo Likert. Se ha encontrado que este nuevo instrumento cuenta con una carga factorial que explica una mayor cantidad de varianza (61.5\%), en comparación con otras adaptaciones del AEQ (Templos, Villalobos, \& Cervera, 2013).

Brown, Golman, Inn y Anderson (1980), en Estados Unidos, entrevistaron adultos; de las trascripciones redactaron los ítems para después pilotear y obtener el $A E Q$, que evalúa las expectativas positivas que se tienen con respecto al consumo de alcohol en seis dimensiones: Cambios positivos globales, Aumento de la sexualidad, Placer físico y social, Asertividad, Incremento del poder y la agresión y Reducción de la tensión, con una escala de respuesta dicotómica.

Desde su creación, el Cuestionario de Expectativas hacia el Alcohol (AEQ), en sus diferentes variaciones, ha sido el más utilizado para indagar sobre las principales expectativas con respecto al consumo de alcohol; fue retomado, por ejemplo, por Mora-Ríos, Natera, Villatoro y Villalvazo (2000), quienes trabajaron con jóvenes mexicanos y obtuvieron un instrumento de 51 ítems, que mide ocho expectativas diferentes: Interacción social, Expresión verbal, Desinhibición, Incremento de la sexualidad, Reducción de la tensión psicológica, Reducción de la tensión física, Cambios psicofisiológicos y Agresividad y sentimientos de poder. Estos investigadores reportaron que las expectativas de interacción social y de reducción de la tensión psicológica fueron los principales predictores del abuso de alcohol entre una muestra de universitarios mexicanos, mientras que las expectativas de incremento de poder, la agresión y el abuso de alcohol, predicen los problemas que, por lo general, se originan por el consumo; esto concuerda con la aceptación que se tiene dentro de la cultura popular y la creencia de que las interacciones sociales serán menos complacientes si no se consume alcohol en exceso (Mora-Ríos, Natera, \& Juárez, 2005).

De igual forma, Camacho (2005), en un estudio con universitarios de la ciudad de Bogotá, utilizó el AEQ (adaptado por Mora-Ríos et al., 2000); observó que una mejor interacción y expresividad verbal, la desinhibición y los cambios psicofisiológicos son las principales expectativas que los jóvenes tienen hacia el consumo de alcohol, ya que les sirve como estrategia de afrontamiento social y reductor del afecto negativo; entre mayor es la frecuencia y la intensidad en el consumo de bebidas alcohólicas mayores serán las expectativas.

Ardila y Herrán (2008), al trabajar con población colombiana, reportaron que la desinhibición y los senti- 
mientos de poder son las principales expectativas asociadas y predictoras del consumo de alcohol. Utilizaron su propia adaptación, el AEQ-III, el cual cuenta con 120 ítems e identifica cuatro factores: Incremento de la expresividad y la sexualidad, Disminución de la tensión física, Disminución de la tensión psicológica y Desinhibición y sentimientos de poder.

Con su adaptación del AEQ-A, que cuenta con 37 ítems, medidos en una escala dicotómica en seis dimensiones, de las cuales cinco hacen referencia a expectativas positivas (relajación y asertividad social, placer físico y social, potenciación de la experiencia social, cambios positivos globales e incremento de la potencia y la agresión) y una a las negativas (impedimento físicos y cognitivos), Peltzer, Lichtenberger, Conde y Cremonte (2017) encontraron, en población Argentina, que las expectativas más relevantes para esta población estuvieron relacionadas con la mejora en el desempeño social, pues entre los adolescentes, el consumo de alcohol suele darse en diversas situaciones sociales, con el fin de facilitar las interacciones; es poco común que los jóvenes hagan uso de la sustancia de forma individual.

La literatura describe varios estudios dirigidos a la adaptación del AEQ, que dan como resultado nuevos instrumentos debido a las grandes modificaciones que sufre el original; es difícil establecer un constructo en general debido a las cuestiones culturales, ya que las expectativas pueden variar de acuerdo con el ambiente sociocultural en el que se desenvuelva la persona (Conde, Lichtenberger, \& Peltzer, 2014; Pelteer et al., 2017).

Las expectativas hacen referencia a las creencias personales que llevan a cada individuo a reaccionar y actuar de determinada forma, en espera de algún resultado (Brown et al., 1980), así como a la relación que se genera entre eventos futuros y sus consecuencias de forma anticipada. Como todo acto conlleva una consecuencia ya sea positiva o negativa, en este caso, si la persona tiene expectativas positivas mayores al ingerir alcohol, sus deseos por consumir aumentarán; al contrario, si tiene más presentes las negativas, su motivación disminuirá (Goldman, Brown, Christanse, \& Smith, 1991). En este sentido, Mora-Ríos et al. (2000), retomando a Brown et al. (1980), describen las creencias positivas que los individuos van formando con respecto a los efectos del alcohol sobre la conducta social y emocional. Las expectativas ayudan a decidir entre beber o no beber alcohol, pues son las consecuencias esperadas del consumo (Reich, Below, \& Goldman, 2010).

Dada la importancia que tienen las expectativas sobre el uso de esta sustancia, resulta imposible no considerarlas, cuando se trata de hacer evaluaciones en las estrategias dirigidas a reducir el consumo excesivo. El Cuestionario de Expectativas hacia el Alcohol (AEQ), adaptado previamente por Mora-Ríos et al. (2000), es un instrumento utilizado en el área de la salud y las adicciones; sin embargo, su última revisión fue realizada hace 17 años, por lo que se vio la pertinencia de revisarlo y hacer modificaciones en las instrucciones, la redacción de los ítems y la escala de respuestas.

En el estudio se partió del Enfoque del Proceso de Acción en favor de la Salud (Schwarzer \& Fuchs, 1999), que hace referencia a los diferentes tipos de cogniciones que participan en la adopción de conductas saludables, como percibirse en riesgo, la autoeficacia y las expectativas de resultado; define las expectativas como las creencias subjetivas que los individuos tienen con respecto a los posibles resultados, mediante afirmaciones del tipo si/luego (Schwarzer, 2008). Con base en esto, se revisaron los reactivos del instrumento y se observó que no reflejaban las creencias en términos de una relación de causalidad; además de que las opciones de respuesta, presentadas en forma dicotómica, reducían la posibilidad de que los estudiantes respondieran el instrumento de una manera más cercana a su situación.

En este documento se reportan las características psicométricas del instrumento mencionado de manera previa, una vez realizadas las modificaciones pertinentes y su aplicación con una muestra de jóvenes universitarios que reportaron consumo de alcohol y fueron evaluados respecto a la frecuencia y la cantidad de consumo, a fin de determinar si, en efecto, quienes tuvieran un mayor puntaje en sus expectativas, también lo tenían respecto al patrón de consumo de alcohol.

\section{MÉTODO}

\section{Diseño del estudio}

Al inicio, se tuvo una muestra total de 4,787 estudiantes de licenciatura. Por medio del muestreo probabilístico aleatorio simple, se obtuvo una muestra de 510 estudiantes (10 por cada ítem del instrumento), con quienes se aplicó el instrumento en una sola ocasión.

\section{Sujetos}

510 estudiantes de licenciatura que cursaban el primer año de estudios universitarios, de las diversas disciplinas existentes en las universidades participantes. 51\% fueron mujeres y $49 \%$, hombres; $98 \%$ de los participantes eran solteros y $2 \%$, casados, vivían en unión libre o eran divorciados. Tenían edades entre los 16 y los 36 años, con una edad promedio de 18.93 años $(D T=1.91)$. 


\section{Sede donde se llevó a cabo la recolección de datos}

El levantamiento de la información se realizó en seis universidades públicas y tres privadas, ubicadas en el estado de Aguascalientes y asociadas al Consejo Interuniversitario Contra las Adicciones (CICA).

\section{Mediciones}

Cuestionario de Expectativas hacia el Alcohol (AEQ), versión adaptada en México (Mora-Ríos et al., 2000). Su objetivo es medir las creencias positivas que el joven tiene con respecto al consumo de alcohol en ocho dimensiones: Interacción social $(a=.79)$, Expresión verbal $(a=.77)$, Desinhibición $(a=.75)$, Incremento de la sexualidad $(a=.78)$, Reducción de la tensión psicológica $(a=.76)$, Reducción de la tensión física $(a=.58)$, Agresividad y sentimientos de poder $(a=.80)$ y Cambios psicofisiológicos $(a=.71)$.

Cuestionario de datos sociodemográficos (INPRFRM, INSP, \& SS, 2011). Evalúa: sexo, edad, estado civil, universidad, carrera, semestre/cuatrimestre. También evalúa el consumo de alcohol alguna vez en la vida, en los últimos 12 meses y su consumo en el último mes.

\section{Procedimiento}

En un primer momento se contactó a los investigadores que adaptaron el $A E Q$, de manera inicial, para población mexicana y se les pidió su autorización para el uso de los reactivos del instrumento, misma que fue otorgada. Por lo que se modificó la redacción de los reactivos y las opciones de respuesta, así como la escala dicotómica, por una de tipo Likert (totalmente en desacuerdo, poco de acuerdo, muy de acuerdo y totalmente de acuerdo), debido a que se retomó el modelo descrito por Schwarzer y Fuchs (1999), que hace referencia al proceso de ejecución de conductas saludables. Siguiendo la lógica de dicho modelo, la redacción de los ítems se hizo considerando una relación de causalidad: "Creo que... si bebo alcohol, entonces..."

Enseguida, los reactivos fueron evaluados por tres jueces expertos en la prevención de las adicciones, quienes hicieron algunas sugerencias a los mismos, entre ellas que se redactara una idea por ítem y que éstas estuvieran generalizadas independientemente del sexo de los estudiantes. Por ello, algunos reactivos se integraron y otros fueron separados, quedando 51 ítems en el instrumento.

Por último, con previa autorización de las autoridades de las diferentes instituciones participantes, los estudiantes respondieron el instrumento en una modalidad electrónica, en los laboratorios de cómputo de sus respectivas escuelas. Al momento de la aplicación se hizo énfasis en que se respondiera lo más sinceramente posible; se garantizó el anonimato y la confidencialidad de la información.

\section{Análisis estadísticos}

Con los datos obtenidos, se realizaron análisis mediante el paquete estadístico SPSS, en su versión 20.0. De manera inicial, se ejecutó un análisis de discriminación de reactivos, a través de la prueba $t$ de student para muestras independientes y mediante un análisis de correlación ítem total.

Los reactivos que pasaron los análisis previos fueron evaluados con la prueba de esfericidad de Barlett y el coeficiente Kaiser-Meyer-Olkin, con el fin de establecer si se justificaba o no el análisis factorial. El análisis factorial exploratorio seleccionado fue el de componentes principales, mediante rotación ortogonal, a través del método varimax, pues se buscaba saber si existía reacomodo y reducción de variables. Finalmente se realizó el análisis factorial confirmatorio de máxima verosimilitud, a través del módulo AMOS del SPSS versión 20.

Para obtener la validez concurrente del instrumento, se realizaron correlaciones entre la frecuencia y la cantidad de consumo de alcohol reportada por los estudiantes, alguna vez en la vida, en el último año, en el último mes y las expectativas evaluadas; se empleó el índice de correlación de Pearson.

\section{Consideraciones éticas}

Cada participante firmó un consentimiento informado, en el cual se explicaba la finalidad del estudio, el resguardo y la protección de sus datos; asimismo, se señalaba la importancia de su participación.

\section{RESULTADOS}

\section{Validez de contenido}

Se obtuvo mediante tres jueces expertos en el tema, quienes revisaron los cambios propuestos en redacción, las instrucciones y las opciones de respuesta del instrumento. Estos realizaron una serie de observaciones, que fueron atendidas hasta alcanzarse el $80 \%$ de acuerdo entre ellos para cada uno de los reactivos. Los cambios sugeridos por los jueces fueron integrar algunos reactivos y separar los que expresaban dos ideas como parte de uno solo. Entre los reactivos que se cambiaron estuvieron "El alcohol disminuye la tensión muscular" y "El tomar hace que las personas se relajen más en situaciones sociales", que fueron integrados en "Me sentiré más 
relajado"; los reactivos "Las mujeres pueden tener orgasmos más fácilmente si han estado bebiendo" y "Los hombres pueden tener orgasmos más fácilmente si han estado bebiendo" se cambiaron por "Tendré un orgasmo más fácilmente". Mientras que los que se separaron fueron: "Me siento una persona más generosa y cariñosa después de haber tomado unas copas" por "Me sentiré una persona más generosa" y "Me sentiré una persona más cariñosa". El reactivo "El beber me hace más franco y terco" también fue separado en "Me sentiré más franco" y "Me sentiré más terco".

\section{Validez factorial}

Por medio del análisis factorial confirmatorio de máxima verosimilitud, se obtuvo la validez factorial del instrumento, mientras que su nivel de confiabilidad se generó a través del índice de consistencia interna Alpha de Cronbach.

La prueba $t$ de student para muestras independientes reveló que los 51 reactivos discriminaron entre el grupo con las calificaciones más altas y el de las calificaciones más bajas. El índice de correlación reactivo calificación total arrojó que todos los reactivos alcanzaron un índice igual o mayor a .20 (Véase la Tabla 1).

La prueba de esfericidad de Barlett y el coeficiente Kaiser-Meyer-Olkin evidenciaron que existía una correlación entre los reactivos. La prueba de Barlett mostró que la matriz de la correlación no era idéntica (1275; $p$ $=<.000$ ). El puntaje Kaiser-Meyer-Olkin alcanzó un valor significativo meritorio (0.97), lo que permitió concluir el análisis. El análisis factorial exploratorio reveló la presencia de siete factores; se conservaron 40 reactivos. El criterio para eliminar reactivos fue que tuvieran valores menores a 40 o que cargaran en dos o más factores; se encontró que tres cargaron en dos factores (22, 47 y 51) y otros ocho obtuvieron una carga factorial menor a .40 $(6,12,14,31,38,42,43$ y 46), por lo que fueron eliminados. Tras esto, y con el fin de probar la estructura factorial obtenida, se llevó a cabo un análisis factorial confirmatorio mediante ecuaciones estructurales, se empleó el paquete estadístico AMOS, lo que resultó en la eliminación de catorce reactivos más $(3,4,19,20,23$, $27,28,29,34,35,37,39,40$ y 44$)$. Los resultados mostraron que el modelo propuesto tuvo un buen ajuste $X^{2}$ $=567.306 \mathrm{gl}=278, p=.000, \mathrm{RMSEA}=.045, \mathrm{NFI}=.91$, $\mathrm{RFI}=.90, \mathrm{RFI}=.95, \mathrm{TLI}=.94, \mathrm{CFI}=.95 \mathrm{Al}$ primer factor se le denominó Desinhibición $(a=.85)$ con cinco ítems; al segundo, Confianza en sí mismo $(a=.83)$ con cinco ítems; al tercero, Reducción de la tensión $(a=81)$ con tres ítems; al cuarto, Relajación $(a=.81)$ con cuatro ítems; al quinto, Incremento de la sexualidad $(a=.77)$ con tres ítems; al sexto, Interacción social $(a=.83)$ con dos ítems; y al séptimo, Cambios psicofisiológicos $(a=.71)$ con cuatro ítems. El instrumento obtuvo una confiabilidad general de .937. La Figura 1 muestra el diagrama de la estructura factorial del instrumento.

\section{Validez de criterio concurrente}

Por último, mediante el índice de correlación de Pearson, se realizaron correlaciones entre la frecuencia y la cantidad de consumo de alcohol reportada por los estudiantes, alguna vez en la vida, en el último año, en el último mes y las expectativas evaluadas.

En un primer momento, se obtuvieron los promedios grupales de los universitarios en cada una de las siete dimensiones. Las expectativas que tuvieron los promedios más altos fueron Desinhibición, Relajación y Confianza en sí mismo, mientras que la que tuvo el promedio más bajo fue Interacción social (Véase Figura 2).

En cuanto al consumo de alcohol se encontró que $85.5 \%$ de los universitarios usó alcohol alguna vez en su vida y $14.5 \%$ no lo hizo. $78.6 \%$ ingirió algún tipo de bebida alcohólica en los últimos 12 meses, $21.2 \%$ no lo hizo y $0.2 \%$ nunca había consumido. $30.2 \%$ tomó de dos a tres veces una bebida de cualquier tipo en el último mes, $29.8 \%$ no había consumido una bebida alcohólica en el último mes, $25.5 \%$ ingirió una vez en el último mes alguna bebida alcohólica y $14.3 \%$ había consumido una bebida alcohólica una o más veces en la última semana. $46.5 \%$ nunca en el último mes tomó cinco o más bebidas alcohólicas, $25.9 \%$ ingirió cinco o más bebidas alcohólicas una vez en el último mes, $18.8 \%$ consumió cinco o más bebidas alcohólicas de dos a tres veces en el último mes y $8.6 \%$ bebió cinco o más bebidas alcohólicas una o más veces en la última semana.

El análisis de correlación de Pearson arrojó una correlación positiva estadísticamente significativa entre los puntajes de la frecuencia de beber una copa, el consumo excesivo de alcohol reportado por los estudiantes en el último mes y las expectativas evaluadas: cuántas veces tomaste en el último mes una cerveza completa o copa de cualquier bebida alcohólica ( $r p=.325, p=.00$ ) y cuántas veces tomaste en el último mes cinco o más cervezas o cualquier bebida alcohólica $(r p=.282, p=.00)$. También se presentaron correlaciones positivas estadísticamente significativas entre haber tomado una cerveza completa o cuba de cualquier bebida alcohólica alguna vez en la vida ( $r p=.182, p=.00$ ) y la frecuencia de haber consumido cualquier tipo de bebida alcohólica en los últimos doce meses ( $r p=.251, p=.00$ ), con los puntajes de las expectativas evaluadas. Es decir, a mayor frecuencia de consumo de una copa de cualquier bebida alcohólica y a mayor frecuencia de consumo excesivo de alcohol, mayores expectativas positivas, en especial en el último mes de consumo. 
Tabla 1

Análisis de discriminación de reactivos

\begin{tabular}{|c|c|c|c|}
\hline \multirow{2}{*}{$\begin{array}{l}\text { Reactivos } \\
\text { CREO QUE SI BEBO ALCOHOL, ENTONCES... }\end{array}$} & \multicolumn{2}{|c|}{$\begin{array}{c}\text { Prueba } t \text { para la } \\
\text { igualdad de medidas }\end{array}$} & \multirow{2}{*}{$\begin{array}{l}\text { Correlación } \\
\text { reactivo- } \\
\text { calificación } \\
\quad \text { total }\end{array}$} \\
\hline & $t$ & $\begin{array}{c}\text { Sig. } \\
\text { (bilateral) }\end{array}$ & \\
\hline 1. Me sentiré acalorado(a). & -7.865 & .000 & .350 \\
\hline 2. Me sentiré bien. & -15.469 & .000 & .613 \\
\hline 3. Las mujeres/hombres serán más sensuales para mí. & -13.845 & .000 & .583 \\
\hline 4. Podré expresar mejor mis sentimientos. & -20.929 & .000 & .698 \\
\hline 5. Me sentiré más autosuficiente. & -13.493 & .000 & .657 \\
\hline 6. No me preocupará lo que los otros piensen de mí. & -15.944 & .000 & .567 \\
\hline 7. Me ayudará a relajarme en situaciones sociales. & -21.603 & .000 & .709 \\
\hline 8. Me sentiré calmado(a) o sereno(a). & -13.595 & .000 & .572 \\
\hline 9. Me sentiré valiente y capaz de pelear. & -14.648 & .000 & .651 \\
\hline 10. Me sentiré más satisfecho(a) conmigo mismo. & -12.834 & .000 & .647 \\
\hline 11. Seré mejor compañero(a) para la gente que bebe conmigo. & -14.524 & .000 & 692 \\
\hline 12. Mis sentimientos de aislamiento y separación disminuirán. & -16.971 & .000 & 679 \\
\hline 13. Las mujeres/hombres serán más amigables. & -18.073 & .000 & .663 \\
\hline 14. Seré mejor amante. & -10.879 & .000 & .572 \\
\hline 15. Me sentiré más relajado. & -16.986 & .000 & .608 \\
\hline 16. Me será más fácil hablar con la gente. & -23.754 & .000 & .750 \\
\hline 17. Estaré de mejor ánimo. & -22.484 & .000 & .711 \\
\hline 18. Tendré un orgasmo más fácilmente. & -10.689 & .000 & .524 \\
\hline 19. Me sentiré una persona más generosa. & -14.785 & .000 & .690 \\
\hline 20. Me sentiré una persona más cariñosa. & -20.823 & .000 & .749 \\
\hline 21. Me sentiré menos tímido(a). & -22.033 & .000 & .717 \\
\hline 22. Me sentiré menos tenso(a) o ansioso(a). & -21.951 & .000 & .729 \\
\hline 23. Me sentiré menos temeroso(a). & -21.852 & .000 & .758 \\
\hline 24. Me sentiré más seguro(a) en una situación, sobre todo si tengo una bebida en la mano. & -14.059 & .000 & .698 \\
\hline 25. Me servirá de anestesia y dejaré de sentir dolor físico. & -12.011 & .000 & .546 \\
\hline 26. Me gustará tener relaciones sexuales. & -12.229 & .000 & .568 \\
\hline 27. Me sentiré más hombre/mujer. & -9.582 & .000 & .560 \\
\hline 28. Me sentiré más romántico(a). & -16.755 & .000 & .697 \\
\hline 29. Me sentiré más despreocupado(a). & -22.959 & .000 & .753 \\
\hline 30. Las reuniones me serán más divertidas. & -22.398 & .000 & .671 \\
\hline 31. Olvidaré más fácilmente los malos momentos. & -18.869 & .000 & .687 \\
\hline 32. Me sentiré excitado(a) sexualmente. & -12.171 & .000 & .597 \\
\hline 33. Dejaré de sentir frío. & -14.999 & .000 & .619 \\
\hline 34. Podré alegar o discutir algo con más fuerza. & -16.514 & .000 & .728 \\
\hline 35. Seré más sociable. & -25.533 & .000 & .764 \\
\hline 36. Mi lado bromista sobresaldrá. & -19.993 & .000 & .707 \\
\hline 37. Me sentiré más franco(a). & -19.454 & .000 & .703 \\
\hline 38. Me sentiré más terco(a). & -14.645 & .000 & .618 \\
\hline 39. Me criticaré menos a mí mismo & -15.925 & .000 & .685 \\
\hline 40. Me será más fácil platicar con personas del sexo opuesto. & -20.573 & .000 & .747 \\
\hline 41. Me sentiré sonrojado(a) y acalorado(a). & -13.670 & .000 & .555 \\
\hline
\end{tabular}


Análisis de discriminación de reactivos (continuación)

\begin{tabular}{|c|c|c|c|}
\hline \multirow{2}{*}{$\begin{array}{l}\text { Reactivos } \\
\text { CREO QUE SI BEBO ALCOHOL, ENTONCES... }\end{array}$} & \multicolumn{2}{|c|}{$\begin{array}{c}\text { Prueba } t \text { para la } \\
\text { igualdad de medidas }\end{array}$} & \multirow{2}{*}{$\begin{array}{l}\text { Correlación } \\
\text { reactivo- } \\
\text { calificación } \\
\text { total }\end{array}$} \\
\hline & $t$ & $\begin{array}{c}\text { Sig. } \\
\text { (bilateral) }\end{array}$ & \\
\hline 42. Me será más fácil recordar historias chistosas o bromas. & -17.083 & .000 & .654 \\
\hline 43. Me sentiré más seguro frente a las personas que tienen posición de autoridad. & -14.654 & .000 & .683 \\
\hline 44. Seré más platicador(a). & -27.246 & .000 & .744 \\
\hline 45. Podré divertirme más en las fiestas. & -21.632 & .000 & .706 \\
\hline 46. Podré dormir mejor. & -11.418 & .000 & 492 \\
\hline 47. Los dolores musculares no me molestarán tanto. & -14.975 & .000 & 678 \\
\hline 48. Me preocuparé menos. & -22.225 & .000 & .727 \\
\hline 49. Me será más fácil actuar impulsivamente o tomar decisiones más rápidamente. & -18.933 & .000 & 683 \\
\hline 50. Me sentiré menos presionado por el tiempo. & -21.046 & .000 & .716 \\
\hline 51. Las cosas serán más chistosas o al menos me reiré más. & -24.774 & .000 & .731 \\
\hline
\end{tabular}

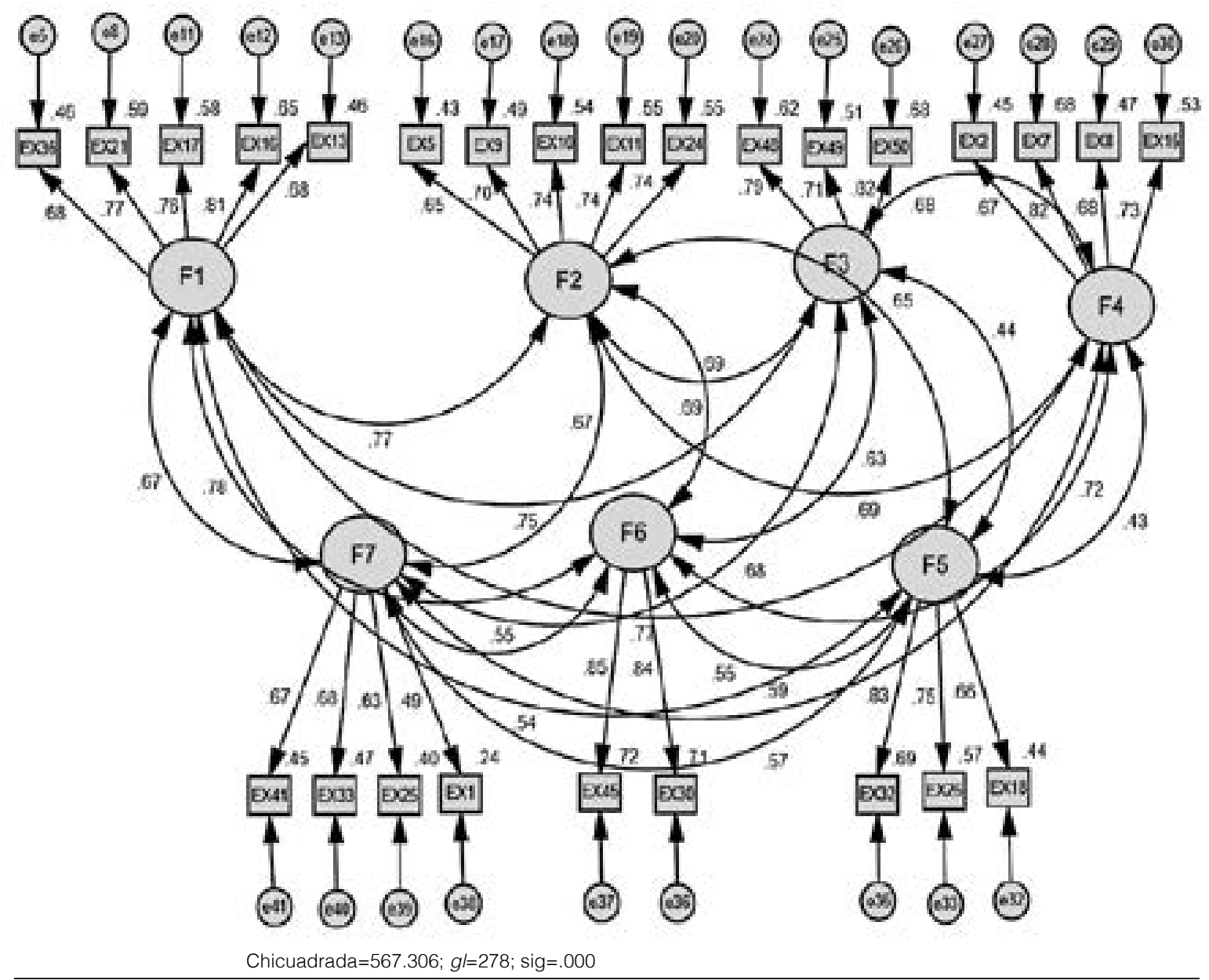

F1= Deshinibición, F2= Confianza en sí mismo, F3= Reducción de la tensión, F4= Relajación, F5= Incremento de la sexualidad,

F6= Interacción social, F7= Cambios psicofisiológicos.

Figura 1. Análisis Factorial Confirmatorio 


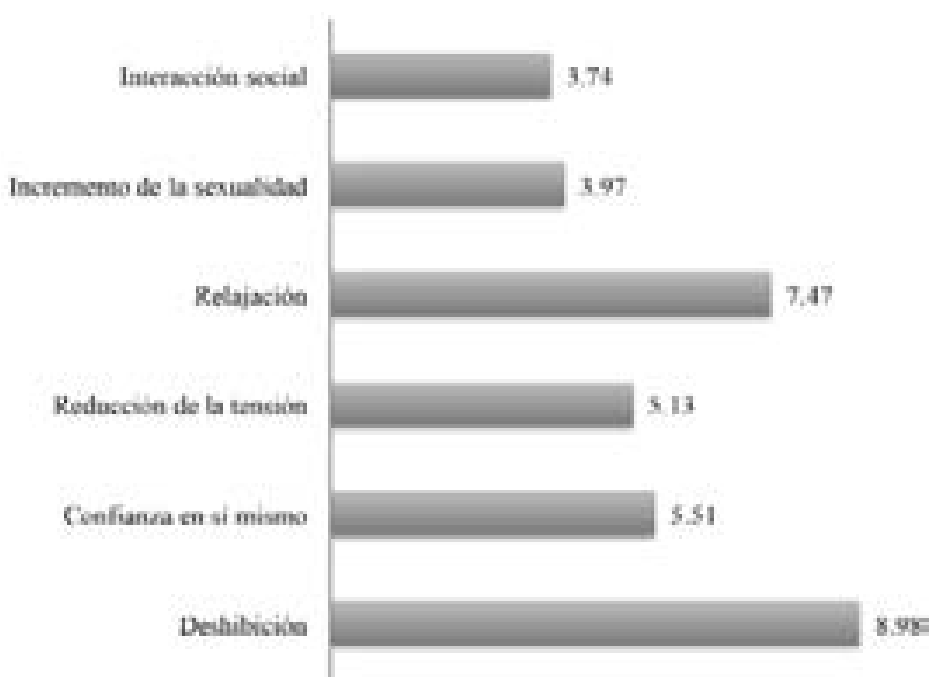

Figura 2. Puntajes promedios de expectativas

Se observaron correlaciones positivas y estadísticamente significativas entre los puntajes de las frecuencia y cantidad de consumo de alcohol reportadas en el último mes por los estudiantes, y los puntajes de las diferentes expectativas. Desinhibición: en el último mes una copa de cualquier bebida ( $r p=.303, p=.00)$ y en el último mes cinco o más bebidas $(r p=.240, p=.00)$. Confianza en sí mismo: en el último mes una copa de cualquier bebida $(r p=.166, p=.00)$ y en el último mes cinco o más bebidas $(r p=.186, p=.00)$. Reducción de la tensión: en el último mes una copa de cualquier bebida $(r p=.202$, $p=.00)$ y en el último mes cinco o más bebidas ( $r p=.130$, $p=.00)$. Incremento de la sexualidad: en el último mes una copa de cualquier bebida $(r p=.181, p=.00)$ y en el último mes cinco o más bebidas $(r p=.195, p=.00)$. Relajación: en el último mes una copa de cualquier bebida $(r p=.361, p=.00)$ y en el último mes cinco o más bebidas ( $r p=.313, p=.00)$. Interacción social: en el último mes una copa de cualquier bebida $(r p=.361, p=.00)$ y en el último mes cinco o más bebidas ( $r p=.339, p=.00$ ). Cambios psicofisiológicos: en el último mes una copa de cualquier bebida $(r p=.153, p=.00)$ y en el último mes cinco o más bebidas $(r p=.130, p=.00)$.

\section{DISCUSIÓN}

La revisión de la validez de constructo del instrumento con base en la definición de expectativas de resultados, derivada del Enfoque del Proceso de Acción en favor de la Salud descrito por Schwarzer y Fuchs (1999), posiblemente tuvo un impacto en el reacomodo de los reactivos en diferentes dimensiones, dando como resultado un número menor de factores, la fusión de algunas de las dimensiones originales y la reducción en el número de ítems. Aunque se conservó el nombre del factor Desinhibición, los reactivos no pertenecían sólo a dicha categoría, pues dentro de esta dimensión se encuentran reactivos que, en la adaptación previa en nuestro país, pertenecían a Expresión verbal, Interacción social, Incremento de la sexualidad, Reducción de la tensión psicológica, Desinhibición y agresividad y Sentimiento de poder. El factor denominado Confianza en sí mismo se fusionó con reactivos pertenecientes, en su mayoría, a la categoría llamada originalmente Agresividad y sentimiento de poder, un reactivo de Incremento de la sexualidad y otro más de Interacción social. El factor denominado Reducción de la tensión fue una fusión de reactivos pertenecientes a las categorías Reducción de la tensión psicológica y Desinhibición. El factor de Relajación resultó de la integración de reactivos de las dimensiones Reducción de la tensión psicológica, Interacción social y Reducción de la tensión física. Cambios psicofisiológicos está integrado por dos reactivos que pertenecían al mismo factor y uno a Reducción de la tensión física. Mientras que Incremento de la sexualidad e Interacción social conservaron reactivos pertenecientes sólo a dichas categorías, por lo cual se decidió conservar los nombres originales.

Los puntajes promedios de los siete factores arrojaron puntuaciones mayores para la Desinhibición, lo cual coincide con lo reportado por García del Castillo (2012) y Ardila y Herrán (2008), quienes explican que los jóvenes usan el alcohol, en espera de que éste les otorgue seguridad y les permita actuar sin miedo. En segundo lugar 
se ubicó la Relajación -que en el estudio de Mora-Ríos et al. (2005) lleva el nombre de Reducción de la tensión psicológica-, como uno de los factores que predicen el abuso en el consumo del alcohol; y en tercero, la Confianza en sí mismo -Agresividad y Sentimientos de Poder, en el mismo estudio de Mora-Ríos et al. (2005)-, que predijo los problemas asociados con el consumo.

Además, los resultados confirman que, a mayor frecuencia de una copa de cualquier bebida alcohólica y el consumo excesivo de alcohol, se tienen mayores expectativas positivas, sobre todo en el último mes, lo que coincide con estudios previos (Camacho et al., 2013). Las dimensiones más sobresalientes en las correlaciones fueron las de Desinhibición, Relajación e Interacción social. De esta forma, es importante trabajar en la prevención del consumo excesivo de alcohol, mediante la promoción de estrategias que impacten en la expresión de las emociones, la mejora de habilidades sociales y el enfrentamiento del estrés.

Sería pertinente, en investigaciones futuras, incluir como variable de estudio la percepción de riesgo; asimismo, se debería trabajar con población de adolescentes y adultos, para confirmar lo que en la literatura se reporta respecto a que la adolescencia y la juventud son periodos en los cuales se tiene una baja percepción de riesgo, por lo que sus creencias se ven centradas en los efectos positivos del beber alcohol (Castaño, García del Castillo, \& Marzo, 2014; García del Castillo, 2012; González-Iglesias, Gómez-Fraguela, Gras, \& Planes, 2014). Es importante realizar esta inclusión porque la percepción de riesgo en la población mexicana ha disminuido con el paso de los años, debido a la aceptación social del alcohol (INPRFM et al. 2012 ; Villatoro et al., 2011).

En conclusión, el estudio permitió hacer mejorías en el AEQ adaptado, dando como resultado un instrumento con un número menor de ítems, con una redacción corta y precisa. El instrumento tiene validez de contenido, factorial y concurrente, y un índice de confiabilidad aceptable para cada una de sus dimensiones, por lo cual se recomienda su uso para acciones encaminadas a la investigación, la prevención y el tratamiento del consumo de alcohol en estudiantes universitarios.

\section{FUENTES DE FINANCIAMIENTO}

Universidad Autónoma de Aguascalientes. Proyecto PIPS14-1, Desarrollo de Estrategias para Promover la Búsqueda de Ayuda en Jóvenes Universitarios que Consumen Alcohol en Exceso.

\section{CONFLICTOS DE INTERÉS}

No hay conflictos de intereses.

\section{AGRADECIMIENTOS}

A la Universidad Autónoma de Aguascalientes, por otorgar el financiamiento para la realización de este estudio; a todos los estudiantes de pregrado y posgrado que contribuyeron con sus observaciones y trabajo de campo; a las escuelas de educación superior asociadas al Consejo Interuniversitario Contra las Adicciones del Estado de Aguascalientes, por las facilidades otorgadas para la ejecución del estudio; y, finalmente, a los investigadores que previamente adaptaron el AEQ en nuestro país, por su consentimento para el uso de los reactivos.

\section{REFERENCIAS}

Aguilar, J., Aguilar, A., \& Valencia, A. (2011). Un modelo explicativo del consumo de alcohol entre estudiantes de licenciatura. Acta de Investigación de Psicología, 1(3), 491-502. Recuperado de http://www.scielo.org.mx/scielo.php?script=sci_arttext\&pid= S2007-48322011000300010

Ardila, F. M., \& Herrán, F. O. (2008). Expectativas en el consumo de alcohol en Bucaramanga, Colombia. Revista Médica de Chile, 136, 73-82. doi: 10.4067/S0034-98872008000100009

Brown, S. A., Goldman, M. S., Inn, A., \& Anderson, L. R. (1980). Expectations of reinforcement from alcohol: their domain and relation to drinking patterns. Journal of Consulting and Clinical Psychology, 48(4), 419-426.

Camacho, A. I. (2005). Consumo de alcohol en universitarios: relación funcional con los factores sociodemográficos, las expectativas y la ansiedad social. Acta Colombiana de Psicología, 13, 91-119. Recuperado de http://www.redalyc.org/articulo.oa?id=79880106

Camacho, L., Mezquita, L., Ibáñez, M. I., Moya, J., Villa, H., Viruela, A. M.,... Ortet, G. (2013). Spanish adaptation of the Expectancy Questionnarie (EQ) about alcohol effects in adolescents. Psicothema, 25(4), 529-535. doi: 10.7334/psicothema2012.354

Castaño, P. G., García del Castillo, J. A., \& Marzo, C. J. (2014). Consumo de alcohol y factores intervinientes en estudiantes universitarios. Revista Cubana de Salud Pública, 4O(1), 1-15. Recuperado de http://www.redalyc.org/articulo.oa?id=21430496006

Conde, K., Lichtenberger, A., \& Peltzer, R. I. (2014).Validez de los cuestionarios de expectativas hacia el consumo de alcohol. In vestigaciones Andinas, 16(28), 881-897. Recuperado de http:// www.redalyc. org/articulo.oa?id $=239030490003$

García del Castillo, J. A. (2012). Concepto de percepción de riesgo y su repercusión en las adicciones. Salud y drogas, 12(2), 133-151. Recuperado de http://www.redalyc.org/articulo.oa?id= 83924965001

Goldman, M. S., Brown, S. A., Christansen, B. A., \& Smith G. T. (1991). Alcoholism and memory: broadening the scope of alcohol-expectancy research. Psychological Bulletin, 110(1), 137146. doi: 10.1037/0033-2909.110.1.137

González-Iglesias, B., Gómez-Fraguela, J. A., Gras, M. E., \& Planes, M. (2014). Búsqueda de sensaciones y consumo de alcohol: el 
papel mediador de la percepción de riesgos y beneficios. Anales de Psicología, 30(3), 1061-1068.

Instituto Nacional de Psiquiatría Ramón de la Fuente Muñiz, Instituto

Nacional de Salud Pública, Comisión Nacional contra las Adicciones, \& Secretaría de Salud. (2017). Encuesta Nacional de Consumo de Drogas, Alcohol y Tabaco 2016-2017: reporte de alcohol. Ciudad de México, México: INPRFM. Recuperado de https://drive.google.com/file/d/1rMIKaWy34GR51sEnBK2-u2q BDK9LA0e/view

Instituto Nacional de Psiquiatría Ramón de la Fuente Muñiz, Instituto Nacional de Salud Pública, \& Secretaría de Salud. (2012). Encuesta Nacional de Adicciones 2011: Reporte de Alcohol. Ciudad de México, México: INPRFM. Recuperado de https://www. gob.mx/cms/uploads/attachment/file/239021/ENA_2011_ Alcohol.pdf

Kumate, R. J. (2003). Percepción de riesgo y consumo de drogas. Recuperado de http://www.salud.gob.mx/unidades/cdi/ documentos/jn2602pers.pdf

Leiva, D. V., Pereira, V., \& Lobo da Costa, M. (2010). Caracterización de estudiantes de la carrera de enfermería sobre consumo de drogas lícitas e ilícitas. Revista Latino-Americana de Enfermagem, 18, 565-572. doi:10.1590/S0104-11692010000700012

Londoño, P. C., \& Valencia, L. S. (2010). Resistencia de la presión de grupo, creencias acerca del consumo y consumo de alcohol en universitarios. Anales de Psicología, 26(1), 27-33. Recuperado de http://www.redalyc.org/articulo.oa?id=16713758004

Monk, L. R., \& Heim, D. (2016). Expectativas relacionadas con el alcohol en adultos y adolescentes: semejanzas y diferencias. Adicciones, 28(1), 35-40.

Mora-Ríos, J., Natera, G., \& Juárez, F. (2005). Expectativas relacionadas con el alcohol en la predicción del abuso en el consumo en jóvenes. Salud Mental, 28(2), 82-90.
Mora-Ríos, J., Natera, G., Villatoro, J., \& Villalvazo, R. (2000). Validez factorial del cuestionario de expectativas hacia el alcohol (AEQ) en estudiantes universitarios. Psicología Conductual, 8(2), 319-328.

Peltzer, R. I., Lichtenberger, A., Conde, K., \& Cremonte, M. (2017). Propiedades psicométricas del Cuestionario de Expectativas hacia el Consumo de Alcohol (AEQ-A) en estudiantes de psicología de Mar del Plata, Argentina. Acta Colombiana de Psicología, 2O(1), 11-21. doi: 10.14718/ACP.2017.20.1.2

Reich, R. R., Below, M. C., \& Goldman, M. S. (2010). Explicit and implicit measures of expectancy and related alcohol cognitions: a meta-analytic comparison. Psychology of Addictive Behaviors, 24, 13-25.

Schwarzer, R. (2008). Modeling health behavior change: how to predict and modify the adoption and maintenance of health behaviors. Applied Psychology, 57(1), 1-29. doi: 10.1111/j.14640597.2007.00325.x

Schwarzer, R., \& Fuchs, R. (1999). Modificación de las conductas de riesgo y adopción de conductas saludables: el rol de las creencias de auto-eficacia. En Bandura, A. (Ed.). Autoeficacia: cómo afrontamos los cambios de la sociedad actual (pp.223244). España: Desclée de Brouwer.

Templos, N. L., Villalobos, G. L, \& Cervera, B. J. (2013). Cuestionario de Expectativas de Resultado de Consumo de Alcohol (CERCA): propiedades psicométricas en pacientes en tratamiento residencial para las adicciones en México. Adicciones, 25(4), 327-332.

Villatoro, J., Gaytán, F., Moreno, M., Gutiérrez, M. L., Oliva, N., Bretón, M.,... Blanco Ordoñez (2011). Tendencias del uso de drogas en la Ciudad de México: encuesta de estudiantes del 2009. Salud Mental, 34(2), 81-94. Recuperado de http://www.scielo.org.mx/ scielo.php?script=sci_arttext\&pid=S0185-33252011000200001 


\section{ANEXO}

Instrumento final

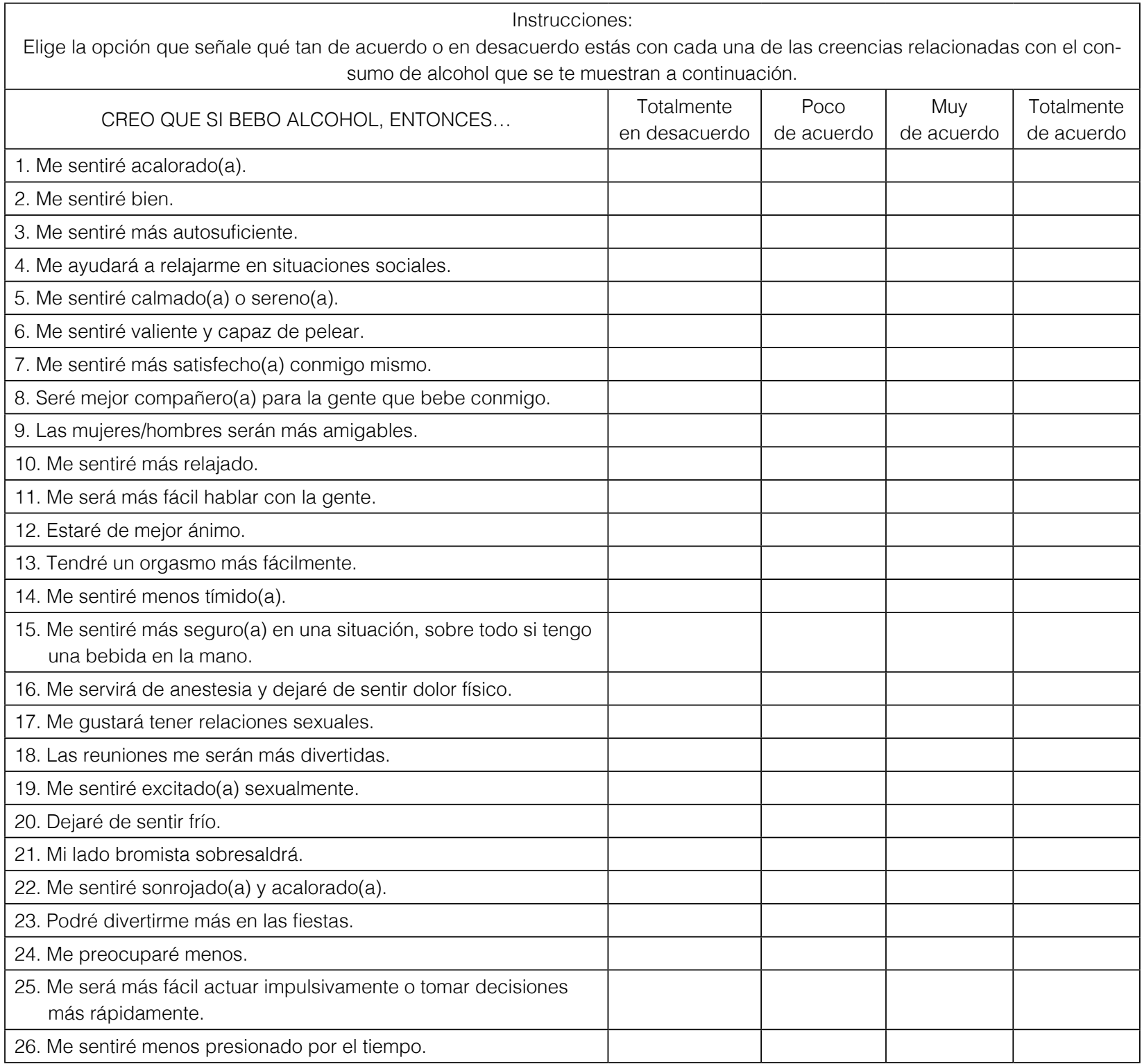

Reactivos por factor

\begin{tabular}{ll}
\hline Factor & Reactivos \\
\hline Desinhibición & $9,11,12,14$ y 21 \\
Confianza en sí mismo & $3,6,7,8$ y 15 \\
Reducción de la tensión & 24,25 y 26 \\
Relajación & $2,4,5$ y 10 \\
Incremento de la sexualidad & 13,17 y 19 \\
Interacción social & 18 y 23 \\
Cambios psicofisiológicos & $1,16,20$ y 22 \\
\hline
\end{tabular}

